\title{
A Segmentation Technique for Flexible Pipes in Deep Underwater Environments
}

\author{
Saulo Pessoa ${ }^{1}$ \\ sap@cin.ufpe.br \\ Vinicius Cesar $^{1}$ \\ vmc@cin.ufpe.br \\ Bernardo Reis ${ }^{1}$ \\ bfrs@cin.ufpe.br \\ Judith Kelner ${ }^{1}$ \\ $\mathrm{jk@cin.ufpe.br}$ \\ Ismael Santos ${ }^{2}$ \\ ismaelh@petrobras.com.br
}

${ }^{1}$ Informatics Center

Federal University of Pernambuco

Recife, Brazil

${ }^{2}$ CENPES

Petrobras,

Rio de Janeiro, Brazil

This paper presents a novel segmentation technique for flexible pipes in deep underwater environments. It consists in finding regions of the pipe denominated vertebrae and has three stages. Firstly, the input image is pre-processed to reduce noise. Secondly, the pre-processed image is binarized by the proposed Multi-level Topological Binarization technique, resulting in an image which highlights (in white) the regions that are potential vertebrae (the blobs). Lastly, the pipe is segmented by finding the best sequence of blobs that fulfills a set of restrictions inherent to a pipe.

For improving the technique robustness, an alternating pattern of white (a vertebra) and black regions is marked on the pipe. Results show that the proposed technique can segment pipes even under harsh conditions such as: low contrast between pipe and background; uneven illumination of the pipe; and high level of noise due to floating particles. In particular, the proposed binarization technique achieves valuable results without any parameter, whereas state-of-the-art techniques did not achieve the same quality even after their parameters were fine-tuned for each condition.

Two blurring filters are used for pre-processing input image: a bilateral filter [5] removes small particles floating around, while preserving the vertebrae's edges; and a Gaussian filter uniformly smooths the images.

The proposed binarization technique finds peaks in the pre-processed image. This is done by "slicing" the image into a number of slices $s_{i, b}$ ( $i$ is its index and $b$ is its level). A tree of slices is then constructed by hierarchically connecting nodes of consecutive levels (Figure 1a). Intuitively speaking, peaks are prominences similar to the first one exhibited in Figure 1b. More formally, a peak is a sequence of nodes denoted by $P=\left\{n_{k}\right\}_{k=1}^{m}=n_{1}, n_{2}, \ldots, n_{m}$, such that: $n_{m}$ is a leaf node of the tree; $n_{k}$, such that $1 \leq k<m$, is the parent node of $n_{k+1} ; n_{1}$ is the only element that has a brother node, or $n_{1}$ is the only element that has a parent with genus greater than zero, or $n_{1}$ is the root node. Nodes $n_{1}$ and $n_{m}$ are respectively named the base and the top of a peak. Given a tree of slices, one has to traverse it from leaves to root in order to find its peaks. For example, Figure 1a contains three peaks: the first one is $P^{\prime}=s_{1,2}, s_{1,3}$; the second is $P^{\prime \prime}=s_{2,2}, s_{2,3}$; and the third is $P^{\prime \prime \prime}=s_{3,2}, s_{3,3}, s_{1,4}$.

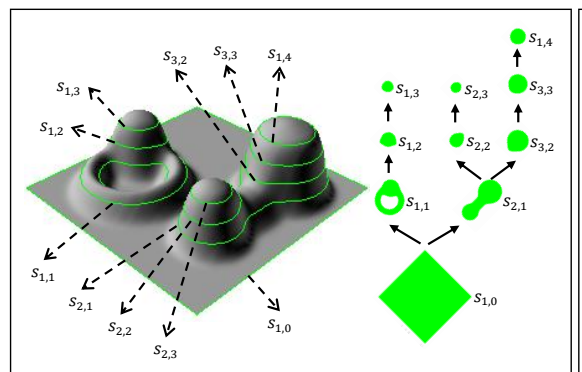

(a)

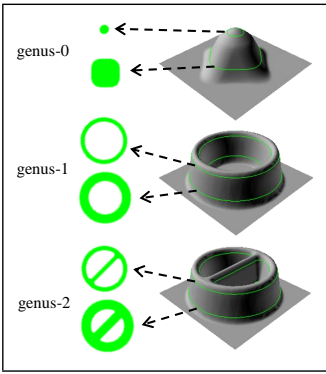

(b)
Figure 1: Surface plot of an image and the respective tree of slices in (a). Topological classification of slices obtained from different surfaces in (b).

The binarization stage produces a set of blobs $B=\left\{b_{i}: i \in\{1, \ldots, n\}\right\}$ (the white regions depicted in Figure 2). The blobs due to the pipe vertebrae are selected by a backtracking algorithm. It searches for the longest chain of blobs that respects restrictions inherent to a pipe. This chain is denoted by a sequence $V=\left\{j_{k}\right\}_{k=1}^{m}=j_{1}, j_{2}, \ldots, j_{m}$, where $j_{k}$ is the index of a blob $b_{i}$. During the search, $b_{i}$ is the current blob being tested to be inserted into $V ; b_{l}$ is the last blob inserted into $V$; and $b_{p}$ is the blob inserted immediately before $b_{l}$.
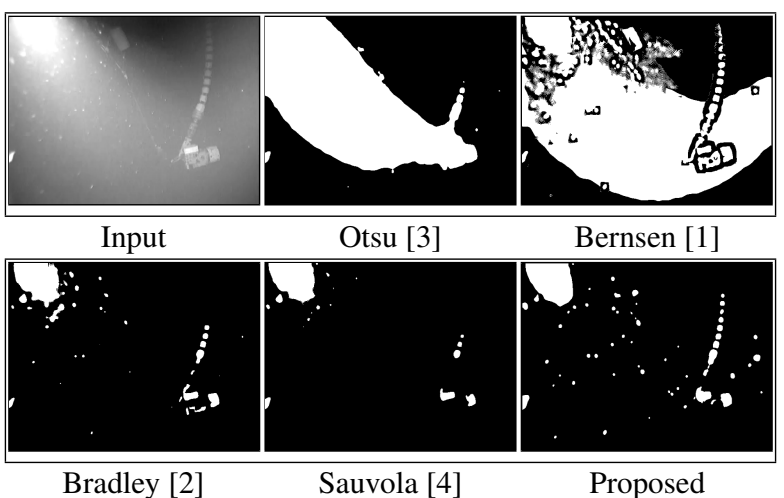

Figure 2: Input image depicting uneven contrast and noise. Results of Otsu, Bernsen, Bradley, and Sauvola binarization algorithms after parameters were fine-tuned. The proposed binarization technique extracts most of the pipe vertebrae without requiring any parameterization.

The first restriction limits the distance between the centroids of $b_{i}$ and $b_{l}$, respectively $c_{i}$ and $c_{l}$ :

$$
d\left(c_{i}, c_{l}\right) \leq d_{\max },
$$

where $d_{\max }$ is the maximum acceptable distance. The second restriction limits the length of the gap between $b_{i}$ and $b_{l}$. This gap is denoted by the line segment $\overline{o_{i} o_{l}}$, where $o_{i}$ is the closest intersection point between the line segment $\overline{c_{i} c_{l}}$ and the contour of $b_{i}\left(o_{l}\right.$ is similarly defined):

$$
d\left(o_{i}, o_{l}\right) \leq g_{\max },
$$

where $g_{\max }$ is the maximum acceptable gap length. The third restriction limits the distance $d\left(o_{i}, c_{i}\right)$, which can be understood as a radius of $b_{i}$. This distance has an upper and lower limit:

$$
\left(\frac{1}{1+v_{\max }}\right) \times d\left(o_{l}, c_{l}\right) \leq d\left(o_{i}, c_{i}\right) \leq\left(1+v_{\max }\right) \times d\left(o_{l}, c_{l}\right),
$$

where $v_{\max } \geq 0$ is a user parameter. The last restriction limits the exterior angle between $\overline{c_{p} c_{l}}$ and $\overline{c_{l} c_{i}}$, which is denoted by $\theta_{i}$. Since three points are required to form an angle, this restriction only applies when there are at least two blobs already inserted into $V$ :

$$
\theta_{i} \leq \theta_{\max }
$$

where $\theta_{\max }$ is a parameter defined by consulting the pipe's specification.

[1] John Bernsen. Dynamic thresholding of grey-level images. In International conference on pattern recognition, pages 1251-1255, 1986.

[2] Derek Bradley and Gerhard Roth. Adaptive thresholding using the integral image. Journal of graphics, gpu, and game tools, 12(2):1321, 2007.

[3] Nobuyuki Otsu. A threshold selection method from gray-level histograms. Automatica, 11(285-296):23-27, 1975.

[4] Jaakko Sauvola and Matti Pietikäinen. Adaptive document image binarization. Pattern recognition, 33(2):225-236, 2000.

[5] Carlo Tomasi and Roberto Manduchi. Bilateral filtering for gray and color images. In Computer Vision, 1998. Sixth International Conference on, pages 839-846. IEEE, 1998. 